\title{
Bilateral Gonadoblastoma Overgrown by Dysgerminoma of the Right Gonad in a Patient with Swyer Syndrome
}

Mohammad ARAFAa, Marwa AI RYIAMIa, Maryam Al SHUKRI b, Ikram BURNEYc, Yasser MAHFOUZ ${ }^{\text {, }}$ Nashwa AL-KINDI ${ }^{\mathrm{e}}$

aDepartment of Pathology, Sultan Qaboos University Hospital, Oman

bDepartment of Obstetrics and Gynecology, Sultan Qaboos University Hospital, Oman

`Department of Medicine (Oncology Unit), Sultan Qaboos University Hospital, Oman

dDepartment of Radiology and Molecular imaging, Sultan Qaboos University Hospital, Oman

eHistopathology Training Program, Oman Medical Specialty Board, Muscat, Oman

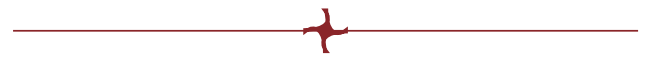

\begin{abstract}
Patients with Swyer syndrome, "XY gonadal dysgenesis", have fibrosed gonads with a significant risk of developing germ cell tumours. During radiological assessment, a 17-year-old female with Swyer syndrome showed mildly enlarged gonads that were removed laparoscopically and proved pathologically to be bilateral gonadoblastomas. In addition, the right sided lesion showed overgrowth by dysgerminoma. The patient was further treated with combination chemotherapy and she was in complete remission for three years.
\end{abstract}

Keywords: gonadoblastoma, dysgerminoma, Swyer syndrome, histopathology, immunohistochemistry.

\section{INTRODUCTION}

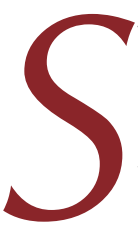
wyer syndrome, also known as XY gonadal dysgenesis, is a type of hypogonadism in a patient whose karyotype is $46 \mathrm{XY}$. It is a rare sexual development disorder that affects approximately 1 in 80,000 live births (1). The affected persons typically show normal female external genitalia. They have functionless fibrosed gonads "streak gonads" and, if left untreated by surgical removal, gonads will progress to develop neoplastic lesions $(1,2)$. In these cases, the typical tumour formation is a germ cell tumour, especially gonadoblastoma ( 1 ).

According to the WHO classification of tumours of the female genital tract, gonadoblastoma is an ovarian germ cell neoplasm that predominantly occurs in phenotypic females with gonadal dysgensis and abnormal karyotype, but 
also, exceptionally, in apparently normal females with a 46 XX karyotype (2). The lesion is usually discovered during evaluation for primary or secondary amenorrhea; however, some are incidentally identified in the form of adnexal calcifications detected during imaging studies $(1,2)$. More than $40 \%$ of gonadoblastomas are bilateral $(2,3)$. The most common karyotypes are $46 \mathrm{XY}$ (Swyer syndrome) and 45X/46XY. Rare patients with a 46 XX karyotype have been reported (2). Pure gonadoblastoma is benign. It is considered as an in situ form of malignant germ cell tumour. The prognosis of tumours with malignant transformation is dependent on the tumour type, size and stage of the secondary malignant component (3).

Dysgerminoma is a primitive germ cell tumour composed of cells without any specific pattern of differentiation. It is the most common malignant germ cell tumour of the ovary, but responsible for only $1-2 \%$ of all malignant ovarian tumours $(2,3)$. It is the most common malignant gonadal tumour in patients with gonadal dysgenesis. It typically arises in a gonadoblastoma in this setting (3).

Herein, we report a case of bilateral ovarian gonadoblastoma overgrown by right sided dysgerminoma in a patient with Swyer syndrome.

\section{CASE REPORT}

A 17-year-old female presented with primary amenorrhea and was diagnosed to have Swyer syndrome. During assessment for prophylactic gonadectomy, ultrasound was performed and revealed small sized uterus but the ovaries were not visualized; therefore, MRI was recommended. Post contrast MRI scan revealed rudimentary uterus and absence of normal ovarian tissues. There were two well defined soft tissue like lesions noticed at the bifurcation of the right common iliac vessels and the other was along the left external iliac artery (Figure 1). These mildly enlarged gonads were resected laparoscopically and sent for histopathological examination. The specimen was received as multiple pieces, measuring in aggregate $4.5 \times 4 \times 0.5 \mathrm{~cm}$ (right side) and one piece measuring $1.5 \times 0.5 \times 0.3 \mathrm{~cm}$ (left side). The entire biopsy material was submitted for routine examination.

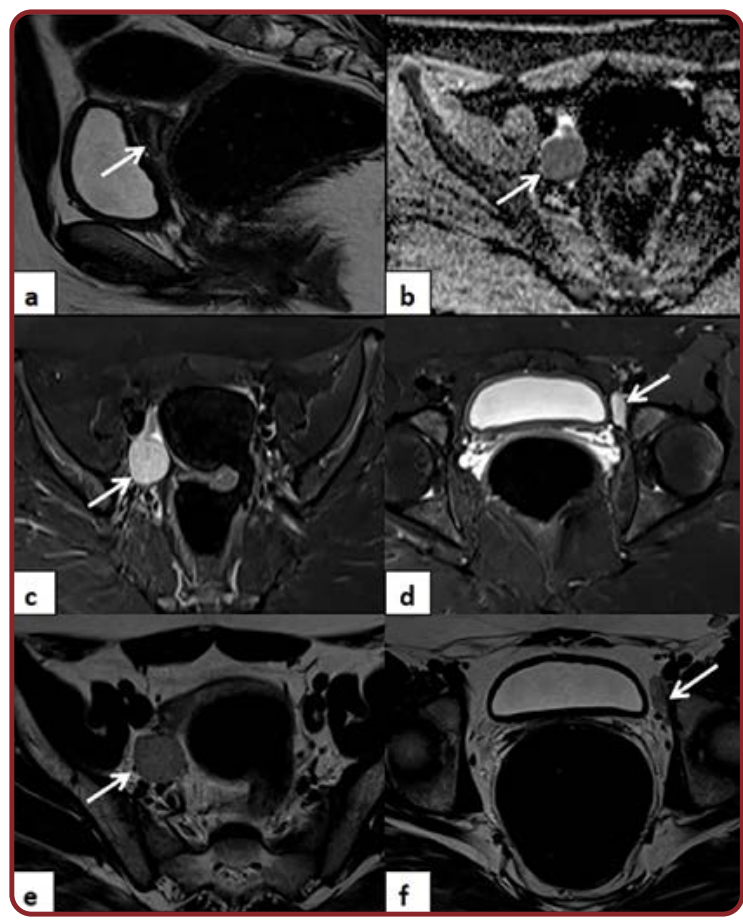

FIGURE 1. Multiple MRI sequences showing two lesions: one at the bifurcation of the right iliac vessels and the other one at the left external iliac region. Sagittal T2WI showing rudimentary uterus (arrow) (a). Axial ADC showing restricted diffusion (arrow) (b). Axial T1 TIRM showing bright signal intensity of gonadal lesions (arrow) (c, d). Axial T2WI showing intermediate signal of gonadal lesions (arrow) (e, f).

Microscopic evaluation of both specimens showed tumour tissues formed of mixed germ cell and sex cord stromal elements. The germ cell component was formed of large cells with abundant cytoplasm, vesicular nuclei with prominent nucleoli and with presence of fibrous septae infiltrated by lymphocytes (dysgerminoma-like). The sex cord component had smaller cells with darker nuclei. Other focal area showed areas with Leydig cell morphology. Hyalinization, calcification and granuloma formations were present. In the right sided specimen, the germ cell component was dominant and showed overgrowth of a frank dysgerminoma. By immunohistochemistry (IHC), the germ cell component was positive for CD117 and placental-like alkaline phosphatase (PLAP). The sex-cord stromal component was positive for inhibin, calretinin and WT1. Representative images of the lesions are illustrated in Figure 2.

The patient was treated with combination chemotherapy (four cycles of etoposide and cis- 


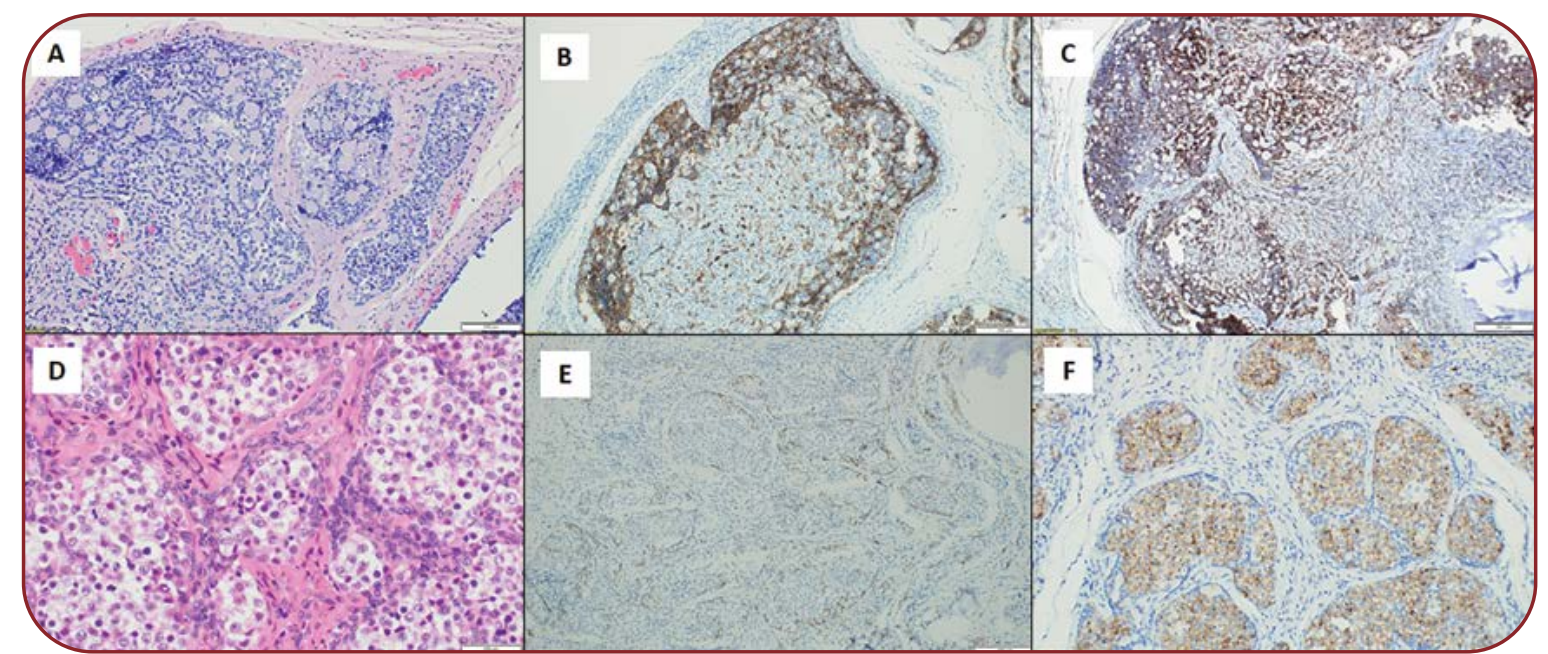

FIG URE 2. Gonadoblastoma component having nests formed of admixture of germ and sex-cord stromal cells by routine H\&E stain (A). CD117 immunohistochemistry (IHC) highlights the germ cell component (B). Inhibin IHC stains the sex-cord component (C). The right sided dysgerminoma overgrowth showing neoplastic cells with round vesicular nuclei and abundant pale cytoplasm, arranged in nests separated by fibrous septae containing lymphocytes (D). Dysgerminoma cells are positive for CD117 (E) and negative for inhibin (F) by IHC

platin). End of treatment scan revealed complete remission and the patient continues to be in a state of complete remission for three years.

\section{DISCUSSION}

W e present a case of ovarian bilateral gonadoblastoma overgrown by dysgerminoma in a patient with Swyer syndrome (46XY gonadal dysgenesis). The patient had female appearance with normal external genitalia, vagina, cervix, uterus and fallopian tubes, and vagina. On the other hand, the ovaries were fibrosed and appeared as gonad streaks. One of the complications of Swyer syndrome is the increased risk of developing tumors in their gonads, namely germ cell tumours $(1,3)$. Dysderminoma is the most common malignant germ cell tumour to develop on top of gonadoblastoma; however, other germ cell tumors have been reported, including immature teratoma, embryonal carcinomas, yolk sac tumor and choriocarcinomas $(2,5)$.

In the current study, the right and left gonads showed the classical diagnostic histopathological features of gonadoblastoma as described (2). In addition, the right sided gonad shows areas of overgrowth of an otherwise typical dysgerminoma (2). Previous studies have described cases that were developing similar germ cell neoplasms $(1,4,5)$. The histopathological diagnosis of gonadoblastoma and dysgerminoma is straightforward in the majority of cases; however, immu- nohistochemistry is required to confirm the diagnosis and exclude other mimics. For example, sex cord tumor with annular tubules (SCTAT) might exhibit similarity to gonadoblastoma in terms of showing similar growth pattern with the presence of calcification (6). In contrast to gonadoblastoma, SCTAT has no germ cell component, which can be confirmed be the lack of CD117 and PLAP immunoreactivity. Moreover, dysgerminoma could have morphological similarity to a variety of ovarian neoplasms. Examples include clear cell carcinoma (CCC) "tubulocystic or papillary architecture, older patients, IHC: SALL4 -/OCT4-/CD117-", small cell carcinoma of hypercalcemic type (HSCC) "smaller tumour cells, pseudofollicular growth pattern, IHC: SALL4 -", Large cell lymphomas "lack of fibrous septae and positive lymphoid IHC markers", Solid pattern of yolk sac tumor (YST) "absence of lymphocytic infiltrate, and IHC: AFP-/glypican 3+/OCT4- phenotype" and solid pattern of embryonal carcinoma "absence of a lymphocytic or granulomatous stromal infiltrate, and IHC: CD30+/CD117- phenotype" (6).

\section{Study limitations}

One limitation of our study is that important immunohistochemical markers (OCT4, SALL 4, FOXL2) for confirmation and typing of germ cell tumours were not performed (2). However, the 
available performed panel (CD117, PLAP, inhibin, calretinin and WT1) was enough to report the final diagnosis with confidence in balance with the histopathological features as assessed using the initial H\&E stained tissue sections. Another limitation was that we were unable to provide an accurate pathological staging because the right sided dysgerminoma specimen was received in pieces. The patient was then subjected to combination chemotherapy. She is in complete remission for three years and is receiving hormonal replacement therapy.

\section{CONCLUSION}

n conclusion, patients with XY gonadal dysgenesis are at high risk of developing germ cell tumours. Their ovaries should be, prophylactically, surgically removed even without the presence of obvious lesions.

Conflicts of interest: none declared.

Financial support: none declared.

Ethical approval: The study followed the ethical guidelines according to the protocol of the Medical Research and Ethics Committee (MREC) of SQU.

Patient consent: Written informed consent and the use of images from patients are not required for the publication.

Acknowledgments: The study was presented, in part, as posters in the BAGP annual meeting and the ESP-IAP congress of 2020.

\section{$\boldsymbol{R}_{\text {EFERENCES }}$}

1. Chand MT, Turner S, Solomon LA, et al. A Case of 45,X/46,XY Mosaicism Presenting as Swyer Syndrome. J Pediatr Adolesc Gynecol 2020;33:577-580.

2. Cheung AN, Ellenson LH, Gilks CB, et al. Tumors of the Ovary. In: Female Genital Tumours, $5^{\text {th }}$ ed, WHO Classification of Tumours Editorial Board, International Agency for Research on Cancer: Lyon, France, 2020, Vol. 4.

3. Prat J, Cao D, Carinelli SG, et al. Germ cell-sex cord-stromal tumors.
WHO Classification of Tumours of Female Reproductive System, $4^{\text {th }}$ Ed, Kurman RJ, Carcangiu ML, Herrington CS, Young RH (eds), International Agency for Research on Cancer Press, Lyon, France, 2014, pp 67-68.

4. Anwar A, Akhtar M, Busby G. Swyer Syndrome: A Case of Dysgerminoma Solely within the Fallopian Tube.

J Pediatr Adolesc Gynecol 2021:S1083-3188(21)00196-0.
5. Raafey MA, Abdulwaasey M, Fatima SS, et al. Bilateral Gonadoblastoma With Dysgerminoma in a Phenotypically Normal Female With 46XX Karyotype: Report of a Rare Case and Literature Review.

Cureus 2020;12:e8990.

6. Maniar KP, Vang R. Germ Cell Tumors of the Ovary. In: Kurman R, Hedrick Ellenson L, Ronnett B (eds). Blaustein's Pathology of the Female Genital Tract. Springer, Cham, 2019. 